\title{
SimPEL: Simulation-based Power Estimation for sequencing studies of Low- prevalence conditions
}

Running Title: Power estimator for rare condition study designs

Lauren Mak ${ }^{1, \#}$, Minghao $\mathrm{Li}^{1, \#}$, Chen $\mathrm{Cao}^{1}$, Paul Gordon ${ }^{1}$, Maja Tarailo-Graovac ${ }^{1}$, Chad

Bousman $^{2,3}$, Pei Wang ${ }^{4}$, and Quan Long ${ }^{*}, 1$

${ }^{1}$ Departments of Biochemistry \& Molecular Biology and Medical Genetics, Alberta Children's

Hospital Research Institute, University of Calgary, AB, Canada

${ }^{2}$ Departments of Medical Genetics, Psychiatry, and Physiology \& Pharmacology, University of Calgary, AB, Canada

${ }^{3}$ Department of Psychiatry, University of Melbourne, Parkville, VIC, Australia

${ }^{4}$ Department of Genetics and Genomics, Icahn School of Medicine at Mount Sinai, NY, USA

\# Joint first author

* To whon correspondence should be addressed

Correspondence:

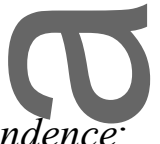

Dr. Quan Long

Room 1173, Health Sciences Centre

Cumming School of Medicine

3330 HospitalDrive NW

Calgary, AB T2N 4N1

This is trum mascript accepted for publication and has undergone full peer review but has not been throw proditing, typesetting, pagination and proofreading process, which may lead to differences hetween this version and the Version of Record. Please cite this article as doi: 10.1002/gepi.2212

This art $\mathrm{a}$ is otected by copyright. All rights reserved. 


\section{quan.long@ucalgary.ca}

\section{ABSTRACT}

Power estimations are important for optimizing genotype-phenotype association study designs. However, existing frameworks are designed for common disorders, and thus ill-suited for the inherent challenges of studies for low-prevalence conditions such as rare diseases and infrequentadverse drug reactions. These challenges include small sample sizes and the need to leverage genetie annotation resources in association analyses for the purpose of ranking potential causal genes. We present SimPEL, a simulation-based program providing power estimations for the design of low-prevalence condition studies. SimPEL integrates the usage of gene annotation resources for association analyses. Customizable parameters, including the penetrance of the putative causal atlele and the employed pathogenic scoring system, allow SimPEL to realistically model a large range of study designs. To demonstrate the effects of various parameters on power, we estimated the power of several simulated designs using SimPEL and captured power trends in agreement with observations from current literature on low-frequency condition studies.

SimPEL, as a tool, provides researchers studying low-frequency conditions with an intuitive and highly flexible avenue for statistical power estimation. The platform-independent, "batteries included" executable and default input files are available at https://github.com/precisionomics/SimPEL.

Keywords. genome-wide sequencing, power estimation, rare disease, adverse drug reactions, association analyses, genetic variant annotation.

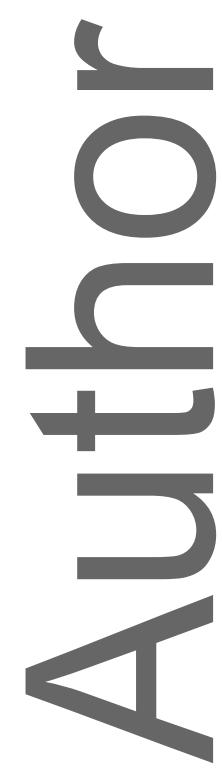

This article is protected by copyright. All rights reserved. 


\section{INTRODUCTION}

As next-generation sequencing became more cost effective over the recent years, genome-wide sequencing has emerged as the standard avenue for identifying causal variants (Adams, Eyans, Henderson, \& Berg, 2016) and has been instrumental in discovering the molecular basis of variants underlying multiple common diseases (Visscher, Brown, McCarthy, \& Yang, 2012). Many researchers are currently embarking on a more difficult objective: elucidating the genetic basis of low-prevalence conditions, such as rare diseases (RD) and infrequent adverse drug reactions (ADR). As an aggregate, RDs affect millions of individuals globally (Boycott, Vanstone, Bulman, \& MacKenzie, 2013). The phenotypic effects range between mild reductions in quality of life, to debilitating or life-threatening, coming at great financial and social costs to public healthcare systems (Boycott et al., 2013; European Organisation for Rare Diseases [EURORDIS], 2005). It is estimated that there are roughly 7,000 distinct RDs, with approximately $80 \%$ having a partial genetic basis (EURORDIS, 2005; Wangler et al., 2017). Research foundations from around the world in the US ("Research Undiagnosed Diseases Network," n.d.), the UK ("NIHR BioResource » Current research,” n.d.), and Canada ("Research - Rare Disease Foundation," n.d.) are focused on tackling RDs, demonstrating the gravity and importance of this issue. ADRs are also placing a significant burden on public healthcare systems: data from around the world show that $3-7 \%$ of all hospitalizations are due to ADRs (Lundkvist \& Jönsson, 2004). Many pharmacogenomic researchers have begun to study ADRs in spite of the generally limited number of cohorts for individual conditions (Giacomini et al., 2016; Pirmohamed, 2014; Urban, 2013).

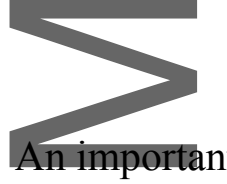
power, which represents the probability of discovering genuine signals. Performing power estimation prior to carrying out a study is the norm for common disease studies, given the wide availability of a variety of models and tools (Lee, Abecasis, Boehnke, \& Lin, 2014; Sham \& Purcell, 2014). From a purely statistical genetics perspective, low-prevalence condition studies are underpowered due to their scale, as available cohorts frequently encompass ten or fewer individuals. In practice, however, the likelihood of success is not as pessimistic as a standard statistical calculation may indicate. Some instances of low-prevalence condition genetic architecture feature high penetrance. The inclusion of this information as an assumption can considerably improve the statistical power of a study design, a term we use to refer to the specific set of parameters that are customizable by the user. Furthermore, functional annotations provided by studies such as the ENCODE Project (ENCODE Project Consortium, 2012) offer

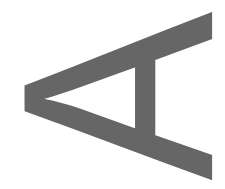

This article is protected by copyright. All rights reserved. 
insight to narrow down the candidate causal gene list. Recently, the Exome Aggregation Consortium and GnomAD Project (Lek et al., 2016) released a large database of exomes and genomes sequenced from individuals who are not affected by a severe early onset disease, providing a comprehensive reference panel of allele frequencies in the "general population". Many of the participants in ExAC are the control samples of population-wide studies of disease subgroups mainly focused on common diseases. A known limitation of ExAC is that clinical (phenotypic) data is unavailable. As an aggregation of exomic allele frequencies, it is not entirely representative of a general 'healthy' population. As such, it is not a perfect population frequency filter, but a serviceable one nonetheless thanks to widespread representation from many different cohorts. Importantly, ExAC allele frequencies are used as points of reference for an unselected low prevalence conditions and have very much strengthened variant annotation $\square$. Furthermore, ExAC is approved for use by the American College of Medical Genetics for use a population database, or a source of variant frequencies of variants large populations. Indeed, part of the lack of data comes from our limited understanding of the effects of rare variants in the 'healthy' population. This population-wide benchmark has improved clinical variant interpretation for the study of low-prevalence conditions by providing indications of the pathogenicity based on the variant's searcity in healthy populations (Bahcall, 2016). Several computational tools calculating for the pathogenicity scores of candidate variants have recently been published (Gulko, Hubisz, Gronau, \& Siepel, 2015; Jagadeesh et al., 2016; Kircher et al., 2014; Ritchie, Dunham, Zeggini, \& Flicek, 2014; Wilfert et al., 2016); the computation process integrates information from both functional annotations and reference panels representing healthy populations. Together with population data on variant frequencies, these tools are helping emerging medical sequencing projects to achieve the goal of producing an 'atlas' of low-prevalence condition pathology, and may in turn bring about therapeutic opportunities (Boycott et al., 2013).

Since the introduction of whole-exome sequencing studies, researchers have carried out rigorous power analyses on large sample-based investigations (Kryukov, Shpunt, Stamatoyannopoulos, \& Sunyaev, 2009). The statistical genetics community has devoted significant efforts toward theoretical and empirical studies (Lee et al., 2014; Sham \& Purcell, 2014) as well as software development; for example, SEQPower was developed to quantify power for common disease study designs (Wang, Li, Lyn Santos-Cortez, Peng, \& Leal, 2014). In contrast, investigators designing low-prevalence condition studies do not have readily available tools to perform the same task. There are several hurdles associated with low-prevalence condition studies. (1) the small sample size is ill-suited for traditional statistical frameworks; and

This article is protected by copyright. All rights reserved. 
(2) modern analytic frameworks integrating annotations and reference panels are statistically difficult to interpret, especially where machine learning techniques are leveraged in generating the pathogenicity scores.

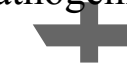

A closed-form derivation to estimate the power of rare condition studies is not currently available and may prove challenging to develop. To meet the present needs of the field, we provide SimPEL, a simulation-based power estimator integrating state-of-the-art variant analysis techniques.

\section{MATERIALS AND METHODS}

\section{Accurate Modelling of the Study Design}

SimPEL assumes a general study design framework where a number of affected cases (subjects) and matched controls (parents, siblings, or unrelated) are assessed through wholegenome or whole-exome sequencing. More complex study designs involving multiple unaffected and affected-offspring are also supported. Familial controls are more frequently used in RD studies, whereas unrelated controls are collected more often in ADR studies for the purpose of matching clinically important features between the cases and controls (e.g. the use of the same medication). These designed studies aim to identify the causal gene(s) of the RD or ADR through the comparison of genetic variants in cases versus controls. SimPEL is intended to evaluate these study designs and provide the statistical power.

SimPEL evaluates statistical power empirically by setting the alpha level, or falsepositive threshold, to the proportion of genes in the 'rank success' list by the total number of genes evaluated. The rank success list of candidate causal genes represents the set of genes associated to an extent warranting experimental follow-up. As such, the size of the list therefore reflects the false discovery rate in the standard sense (Figure 1). The search space may be as broad as the set of all genes or a subset composed of gene candidates, reflecting the a priori knowledge of the focal condition.

To accurately model low-prevalence condition studies, SimPEL takes a variety of customizable parameters as inputs. The software is highly flexible in terms of study design

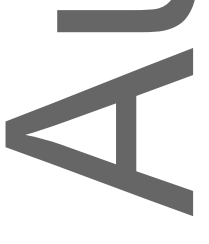

This article is protected by copyright. All rights reserved. 
inputs: key specifications related to the study designed to investigate a condition as well as the condition in question (e.g. sample size of the study, number of causal genes for the condition, the prevalence of the condition in the population, and the penetrance of the causal alleles) are all able to be assigned a specific value by the user or fall back to a default value as determined by the progran. Penetrance and prevalence can be set as fixed values for each round of simulation, or randomly selected from a normal distribution in every round of simulation for instances of single or multiple causal variants. Since the expression of many biological traits are influenced by the environment, or a (epi)genetic-environmental interaction, the penetrance may also be affected by an optional GxE component. The expected MAF of a variant in a general healthy population may be an indicator of penetrance, and its effects can also be modeled with the inclusion of an optional weighting component. SimPEL is a command line software in which the parameters are specified in the initial command to run the program. SimPEL, in the output report, will determine the likelihood of finding the true causal gene of the condition (power) through simulating the study many times based on the particular study design as described by user-entered values. For the complete list of customizable parameters and detailed instructions for using SimPEL, please refer to the User's Manual.

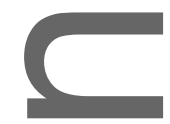

To estimate the statistical power of a study design described by user-designated parameters. SimPEL performs multiple rounds of simulations with the same parameters, using different samples and causal genes generated from the input genotype and causal gene pools, respectively. In each round of simulation, SimPEL first simulates one or multiple causal variants, taking into consideration the causal variant's low frequency in healthy populations and its pathogenicity score. In accordance with user-specified parameters, including the variant's expected prevalence and penetrance, a number of individuals in the template genotype pool [e.g. genomes from the 1000 Genomes Project (1000 Genomes Project Consortium et al., 2015)] will be mutated and thus designated as affected. Sampling from real human genomes in the 1000 Genomes reference panel implicitly incorporates population genetics and linkage structures not present in simulated genomes. For example, if a common variant caused the rare disease, then the power calculated would rightfully be extremely low due to the variant's prevalence in the healthy population. Conversely, if a rare variant caused the rare disease, then the power calculated would be high as the variant would not be found in the healthy population. The mutations are noyel in the sense that they are not present in the original genomes sampled, but they are generated at known random sites that may or may not be well annotated. Matching parental (single-parent or parent-child trio setups), (unaffected) sibling, or unrelated controls are

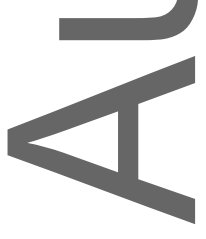

This article is protected by copyright. All rights reserved. 
then sampled accordingly. For example, consider a study design incorporating nine unrelated cases with different family structures. If the parameters designating the number of matched single-parent, both-parent, and sibling controls are set to 4, 3, and 2 respectively, then 4 random cases of the total patient sample will have a single parent as a matched control, 3 cases will have both parents (a 'trio') as matched controls, and 2 random cases will have siblings as matched controls. Emphasis is placed on variants present in cases, but not present in controls, henceforth referred to as "case-specific variants". Following identification, case-specific variants are consequently weighted by pathogenicity scores provided by an annotation tool such as M-CAP or CADD (Jagadeesh et al., 2016; Kircher et al., 2014). Each mutated gene is finally given an overall score to indicate likelihood of causality and an allele frequency reference panel (Campbell \& Rudan, 2002) is applied to the simulated data so as to prioritize a list of genes of user-defined length, sorted and ranked by the overall score. The power is assessed as the proportion of simulations in which the causal gene(s) is included in the shortlist of top genes, as ranked by the overall score (Figure 1). Users are able to set the false positive rate through specifying the length of the top candidate causal gene shortlist, with "success" being defined as inclusion in the list (Figure 1). This procedure is consistent with standard practice in lowprevalence condition studies (Boycott et al., 2013; Cooper \& Shendure, 2011; MacArthur et al., 2014). Detailed descriptions of the genotype and phenotype simulations, as well as the analytic framework, are in the Supplementary Materials and Methods.

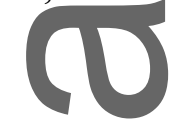

\section{Data Output Format:}

Results of the power estimation procedure for the proposed study design are reported in a text file. The file's header contains a summary of all user-specified parameter values, including all mandatory parameters and any optional parameters specified in the input command. Summary information from each of the simulations is reported in sequential order. For each round of simulation, the information being reported includes the simulated causal gene(s), the generated variants and their locations, the rank of the simulated causal gene in the final shortlist of top genes, and the overall score used to determine its rank in the shortlist. The variants are presented in single lines and display the template genotype ID, the ethnic group, the gender, the location of the variant, the substituted base, and the pathogenicity score of the substitution all separated by a single forward slash (“/”). The power of the simulated study for each heterogeneity status is displayed at the tail end of the file as a rational number between 0.0 and 1.0 along with the program runtime in seconds. For example, should the simulated condition have three causal genes as specified by the input parameter, the powers of nominating at least 1 , at least 2 , and all

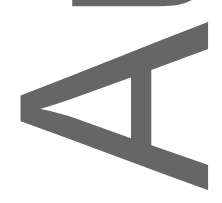

This article is protected by copyright. All rights reserved. 
3 genes to the rank success list will be generated. A power of 1.0 indicates inclusion of the simulated causal gene in the rank success list in every round of simulation and analysis. The file is constantly updated as SimPEL runs. As such, generated information is retained within the output fite in the event of early termination of the process.

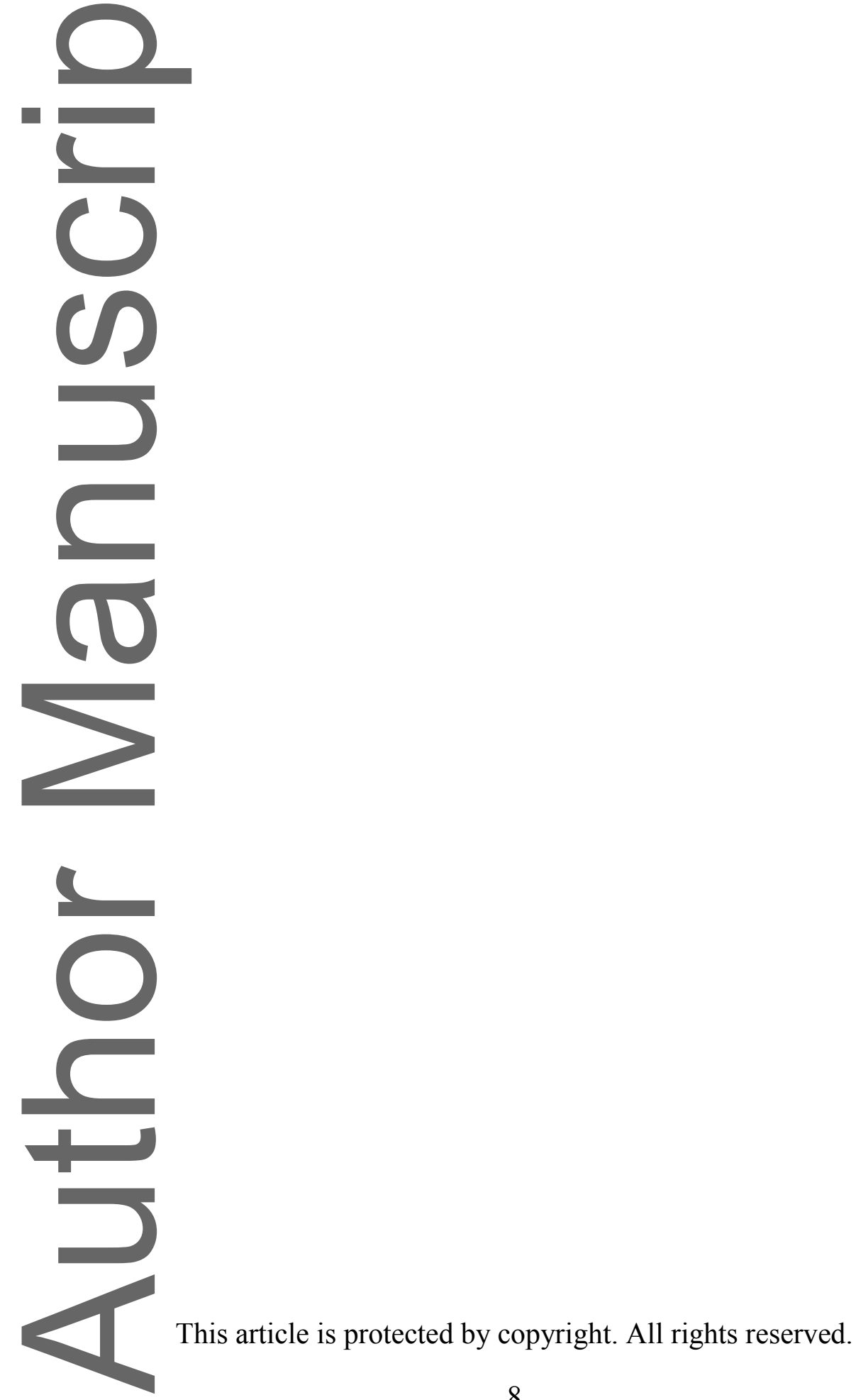




\section{RESULTS}

\section{Customizable Parameters}

By providing highly customizable parameters, SimPEL can be flexibly applied to a variety of study designs. Several of them are listed in Table I. Toggling the parameters to simulate arange of expected study conditions can provide a thorough summary of the relative contributions of each parameter. For example, in situations where the sample size is sufficiently "large," reduced penetrance at a gene in question may decrease the power less than a similar increase in locus heterogeneity, but not as much as the elimination of matched parental controls. The parameters fall into three categories. First, simple parameters related to the study design are incorporated, such as how many cases are paired with matched familial (parent or sibling) controls. Second, parameters relating to the genetic architecture permit the incorporation of user expectations with regards to the impact of variants on the phenotype. Factors include (1) the penetrance of the causal alleles; (2) whether the condition is caused by a single gene or multiple genes; (3) whether a list of known candidate causal genes is available; and (4) the expected frequency of the causal allele(s) within the general population (i.e. filtering for the minor allele frequency (MAF) cutoff in ExAC). Third, analytic parameters, such as pathogenicity score cutoffs, are fully customizable. In addition to the above, SimPEL also allows the user to specify a confidence interval for the scores calculated by standard tools, lending itself to cases where the estimation of pathogenicity may not genuinely reflect the magnitude of the variants' effect. The full list of the parameters and their descriptions is available in the User's Manual.

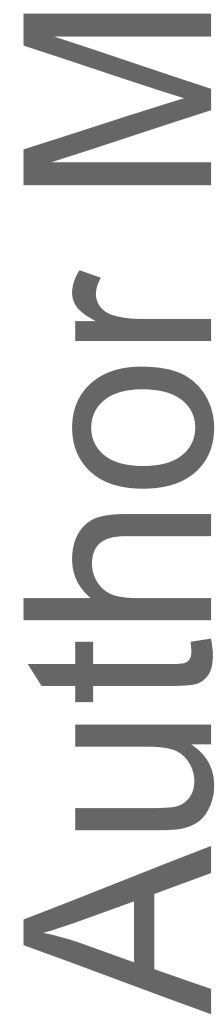

This article is protected by copyright. All rights reserved. 


\section{Insights Revealed by Simulations}

SimPEL utilizes a specific set of input files for the purpose of simulations. Three major inputs are the population variant frequency, MAFs, and pathogenicity scores. For these inputs, the default files included with the program are from the 1000 Genomes Project (1000 Genomes Project Consortium et al., 2015), the Exome Aggregation Consortium (Lek et al., 2016), and MCAP (Jagadeesh et al., 2016), respectively. Population variant frequencies genotypes serve as a template for the generated individuals in simulations. MAFs allow for the identification of minor allele counts in healthy populations. Lastly, pathogenicity scores indicate the disease causal likelihood of generated variants in the simulations.

To demonstrate the utility of SimPEL, simulations were carried out using autosomal recessive genes as the input causal gene pools. Compound heterozygosity was also flagged for the purpose of these sample simulations. The results show that power increases proportionally with the sample size (Figure 2A), the minimum pathogenicity score (Figure 2B), the penetrance (Figure 2C), and the number of matched parental controls (Figure 3), although a plateau is reached after a certain point (specific to the parameter) and diminishing returns are observed in a logarithmic fashion. Increasing the penetrance appears to have a lower incremental effect on power in comparison with sample size and minimum pathogenicity score. The statistical power expected when identifying causal genes is also reduced with respect to increasing the heterogeneity of the simulated disease, illustrating the difficulty in elucidating multifactorial inheritance disorders. Overall, increases in sample size have the greatest effect on power increase of the three parameters across different heterogeneity statuses. With regards to the number of matched controls, the estimated power spikes rapidly beyond the presence of five controls (Figure 3). Parents represent the best genetic architecture for controls in case-control pairings, and a single parental control within a collection of pairings elevate the power to prioritize the single causal gene (with the assumption of no heterogeneity) of a disease to 1 .

\section{Efficient Implementation}

SimPEL is implemented as an extension to our established JAWAMix5 tool (Long et al., 2013), an HDF5-based Java implementation for association mapping. As Java is platform independent and the software comes "batteries-included," there is no specific installation process required and the program is ready for immediate use. Taking advantage of its ability to store

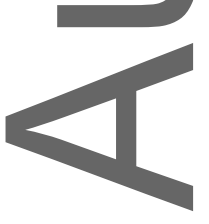

This article is protected by copyright. All rights reserved. 
large genomic data files on disk, but access them as though they are in memory, SimPEL is scalable to very large genome pools while consuming little memory. Computational resources are largely consumed through reading annotation files and comparing the genotypes of cases and controls. A run-time of 60 minutes can be expected given the inclusion of a gene pool consisting of several hundred genes, extending to upwards of 12-24 hours upon inclusion of all human genes. Formore information on how to utilize SimPEL and specific information on all parameters and inputs, please refer to the User's Manual.

\section{DISCUSSION}

We provide for the first time a tool to address the issue of underpowered study designs for low-prevalence condition studies. Our results are in line with known power trends (Gulko et al., 2015) and exhibit the practical usage of SimPEL. In the field of genomic sequencing studies, research foundations funding low-prevalence condition etiology studies are becoming more prevalent. The onus is on interested researchers to provide rational study designs in the face of implicitly small number of affected cases ("NIHR BioResource » Current research," no date, "Research-Rare Disease Foundation," no date). This currently poses a significant challenge for interested researchers, as the customary measure of power cannot be calculated analytically using existing tools for common diseases. SimPEL is a timely tool that addresses this need.

\section{(1)}

A limitation of SimPEL is that, in practice, some of the key parameters, e.g., penetrance, are unknown to researchers. If our default values are not applicable to the study design at hand, researchers may run SimPEL multiple times using different likely values and use the average of the outcomes as the expected power. Another limitation that exists within SimPEL's analytical approach is the specific annotation tools used. For example, some have suggested that CADD scores may not be particularly effective in predicting deleteriousness with regards to non-coding variants (Mather et al., 2016). Mather et al. (2016) state that they could not determine causal rare intronic variants despite significant differences in assigned annotation scores between common and rare SNVs, identifying in silico annotation tools as an area of improvement for future clinical application of GWAS data. Fortunately, given SimPEL's flexibility with regards to inputs, scores generated from a different annotation tool can easily be applied within the program. This demonstrates SimPEL's potential longevity and applicability to revised annotation tools in the future. Users may also choose to indicate their confidence in the scoring system of their selected annotation tool through specifying a value for the confidence parameter.

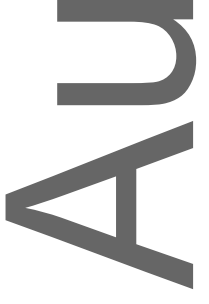

This article is protected by copyright. All rights reserved. 
To determine causality, experimental validation on variants discovered through genomic sequencing is essential, especially for low-prevalence conditions. For studies involving only a handful of cases, it may not be possible to independently replicate the results, and by extension make a strong statistical case for association. As such, integrating the results of statistical and experimental approaches as well as context in the form of patient medical history and familial history are required to establish causality.

SimPEL is the first tool to provide quantitative estimates of power in accordance to the study design and key underlying assumptions regarding the genetic architecture of a focal lowprevalence condition. Given the complexity of the annotations, analysis, and the heterogeneity of different diseases, we expect that simulations will remain essential even as theoretical understanding of statistical methods for low-prevalence conditions improves. Therefore, SimPEL's method of estimating power in the evolving field of low-prevalence condition study has longevity. In addition, SimPEL can be easily updated to accommodate for new innovations, such as improved ad hoc annotation tools or theoretical derivations along the lines of Lee et al. (2014). To facilitate reproducibility in other statistical power estimation tools, users also can use output simulation level variants and overall scores to compare different power estimates. Supplementary information is available online. All example input and output files, as well as a detailed User's Manual, can be found on GitHub at https://github.com/precisionomics/SimPEL. Before preparing their own data, users can interact with sample data to test SimPEL's power estimation capabilities.

\section{ACKNOWLEDGEMENTS}

We would like to express our gratitude towards Dr. Kym Boycott for her insightful comments. This work is supported by the Rare Disease Foundation and BC Children's Hospital Foundation, University of Calgary Startup and URGC Seed Grants, Canada Foundation for Innovation, NSERC Discovery Grant (Q.L.) and Alberta Children's Hospital Research Institute graduate studentship (L.M.).

The authors declare no conflict of interest.

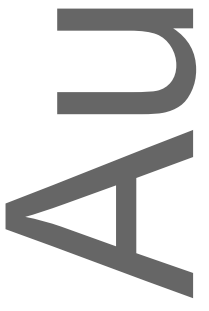

This article is protected by copyright. All rights reserved. 


\section{REFERENCES}

1000 Genomes Project Consortium, Auton, A., Brooks, L. D., Durbin, R. M., Garrison, E. P., Kang, H. M. ... Abecasis, G. R. (2015). A global reference for human genetic variation. Nature, 526(7571), 68-74. https://doi.org/10.1038/nature15393

Adams, M. C., Evans, J. P., Henderson, G. E., \& Berg, J. S. (2016). The promise and peril of genomic sereening in the general population. Genetics in Medicine, 18(6), 593-599. https://doi.org/10.1038/gim.2015.136

Bahcall, O.G. (2016). Genetic variation: ExAC boosts clinical variant interpretation in rare diseases. Nature Reviews Genetics, 17(10), 584-584. https://doi.org/10.1038/nrg.2016.121

Boycott, K. M. Vanstone, M. R., Bulman, D. E., \& MacKenzie, A. E. (2013). Rare-disease genetics in the era of next-generation sequencing: discovery to translation. Nature Reviews Genetics, 14(10), 681-691. https://doi.org/10.1038/nrg3555

Campbell,H., \& Rudan, I. (2002). Interpretation of genetic association studies in complex disease. The Pharmacogenomics Journal, 2(6), 349-360. http:/dx.doi.org/10.1038/sj.tpj.6500132

Cooper, G M., \&Shendure, J. (2011). Needles in stacks of needles: finding disease-causal variants in a wealth of genomic data. Nature Reviews Genetics, 12(9), 628-640. https://dol.org/10.1038/nrg3046

ENCODE Project Consortium. (2012). An integrated encyclopedia of DNA elements in the human genome. Nature, 489(7414), 57-74. https://doi.org/10.1038/nature11247

European Organisation for Rare Diseases. (2005). Rare Diseases: understanding this Public Health Priority. October, (November), 1-14. Retrieved from $\mathrm{http} / / \mathrm{www}$.eurordis.org/IMG/pdf/princeps_document-EN.pdf

Giacomini, K. M. Yee, S. W., Mushiroda, T., Weinshilboum, R. M., Ratain, M. J., \& Kubo, M. (2016). Genome-wide association studies of drug response and toxicity: An opportunity for genome medicine. Nature Reviews Drug Discovery. https://doi.org/10.1038/nrd.2016.234

Gulko,B., Hubisz, M. J., Gronau, I., \& Siepel, A. (2015). A method for calculating probabilities of fitness consequences for point mutations across the human genome. Nature Genetics, 47(3), 276-283. https://doi.org/10.1038/ng.3196

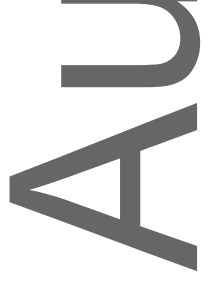

This article is protected by copyright. All rights reserved. 
Jagadeesh, K. A., Wenger, A. M., Berger, M. J., Guturu, H., Stenson, P. D., Cooper, D. N., ... Bejerano, G. (2016). M-CAP eliminates a majority of variants of uncertain significance in clinical exomes at high sensitivity. Nature Genetics, 48(12), 1581-1586. https://doi.org/10.1038/ng.3703

Kircher, M., Witten, D. M., Jain, P., O’Roak, B. J., Cooper, G. M., \& Shendure, J. (2014). A general framework for estimating the relative pathogenicity of human genetic variants. Nature Genetics, 46(3), 310-315. https://doi.org/10.1038/ng.2892

Kryukov, G. V, Shpunt, A., Stamatoyannopoulos, J. a, \& Sunyaev, S. R. (2009). Power of deep, all-exon resequencing for discovery of human trait genes. Proceedings of the National Academy of Sciences of the United States of America, 106(10), 3871-3876. https://doi.org/10.1073/pnas.0812824106

Lee, S., Abecasis, G. R., Boehnke, M., \& Lin, X. (2014). Rare-variant association analysis: Study designs and statistical tests. American Journal of Human Genetics. https://doi.org/10.1016/j.ajhg.2014.06.009

Lek, M., Karczewski, K. J., Minikel, E. V., Samocha, K. E., Banks, E., Fennell, T., ... MacArthur, D. G. (2016). Analysis of protein-coding genetic variation in 60,706 humans. Nature, 536(7616), 285-291. https://doi.org/10.1038/nature19057

Long, Q., Zhang, Q., Vilhjalmsson, B. J., Forai, P., Seren, Ü., \& Nordborg, M. (2013).

JAWAMix 5: an out-of-core HDF5-based java implementation of whole-genome association studies using mixed models. Bioinformatics, 29(9), 1220-1222.

https:/doi.org/10.1093/bioinformatics/btt122

Lundkvist, J., \& Jönsson, B. (2004). Pharmacoeconomics of adverse drug reactions. Fundamental \& Clinical Pharmacology, 18(3), 275-80. https://doi.org/10.1111/j.14728206.2004.00239.x

MacArthur,D. G., Manolio, T. A., Dimmock, D. P., Rehm, H. L., Shendure, J., Abecasis, G. R., ... Gunter, C. (2014). Guidelines for investigating causality of sequence variants in human disease. Nature, 508(7497), 469-476. http://dx.doi.org/10.1038/nature13127

Mathen, C. A., Mooney, S. D., Salipante, S. J., Scroggins, S., Wu, D., Pritchard, C. C., \& Shirts, B. H. (2016), CADD score has limited clinical validity for the identification of pathogenic variants in noncoding regions in a hereditary cancer panel. Genetics in Medicine, 18(12), 1269-1275. https://doi.org/10.1038/gim.2016.44

This article is protected by copyright. All rights reserved. 
NIHR BioResource » Current research. (n.d.). Retrieved October 23, 2017, from https://bioresource.nihr.ac.uk/rare-diseases/study-themes/

Pirmohamed, M.(2014). Personalized Pharmacogenomics: Predicting Efficacy and Adverse Drug Reactions. Annual Review of Genomics and Human Genetics, 15(1), 349-370. https:/doi.org/10.1146/annurev-genom-090413-025419

Research - Rare Disease Foundation. (n.d.). Retrieved October 23, 2017, from https://www.rarediseasefoundation.org/research

Research - Undiagnosed Diseases Network. (n.d.). Retrieved October 24, 2017, from https:/undiagnosed.hms.harvard.edu/research/

Ritchie, G.R.S., Dunham, I., Zeggini, E., \& Flicek, P. (2014). Functional annotation of noncoding sequence variants. Nature Methods, 11(3), 294-296. https://doi.org/10.1038/nmeth.2832

Sham, P. C., \& Purcell, S. M. (2014). Statistical power and significance testing in large-scale genetic studies. Nature Reviews Genetics, 15(5), 335-346. https://doi.org/10.1038/nrg3706

Urban, T. J. (2013). Whole-genome sequencing in pharmacogenetics. Pharmacogenomics, 14(4), 345-348. https://doi.org/10.2217/pgs.12.211

Visscher, P.M., Brown, M. A., McCarthy, M. I., \& Yang, J. (2012). Five Years of GWAS Discovery. American Journal of Human Genetics, 90(1), 7-24.

https://doi.org/10.1016/j.ajhg.2011.11.029

Wang, G. F., Li, B., Lyn Santos-Cortez, R. P., Peng, B., \& Leal, S. M. (2014). Power analysis and sample size estimation for sequence-based association studies. Bioinformatics, 30(16), 2377-2378. https://doi.org/10.1093/bioinformatics/btu296

Wangler, M. F., Yamamoto, S., Chao, H.-T., Posey, J. E., Westerfield, M., Postlethwait, J., ... Bellen, H. J (2017). Model Organisms Facilitate Rare Disease Diagnosis and Therapeutic Research.Genetics, 207(1), 9-27. https://doi.org/10.1534/genetics.117.203067

Wilfert, A. B., Chao, K. R., Kaushal, M., Jain, S., Zöllner, S., Adams, D. R., \& Conrad, D. F. (2016). Genome-wide significance testing of variation from single case exomes. Nature Genefics, 48(12), 1455-1461. https://doi.org/10.1038/ng.3697

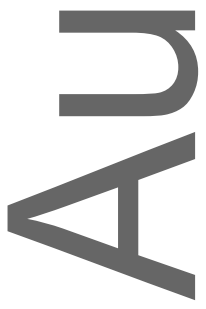

This article is protected by copyright. All rights reserved. 


\section{FIGURE LEGENDS}

Figure 1. Visualization of SimPEL's power estimation step.

Each gradient-bar represents the genes prioritized by their overall score in increasing order after each round of simulation. The number of top candidate genes is fixed by the user, and represented by the solid black line. Each of the grey lines represents the simulated causal gene. If the simulated eausal gene is prioritized to the list of top candidate genes, then the round is considered a success. Of the 8 rounds depicted, all except the last were success. Thus, the power of the study design estimated by these 8 simulations is $7 / 8=0.875$.
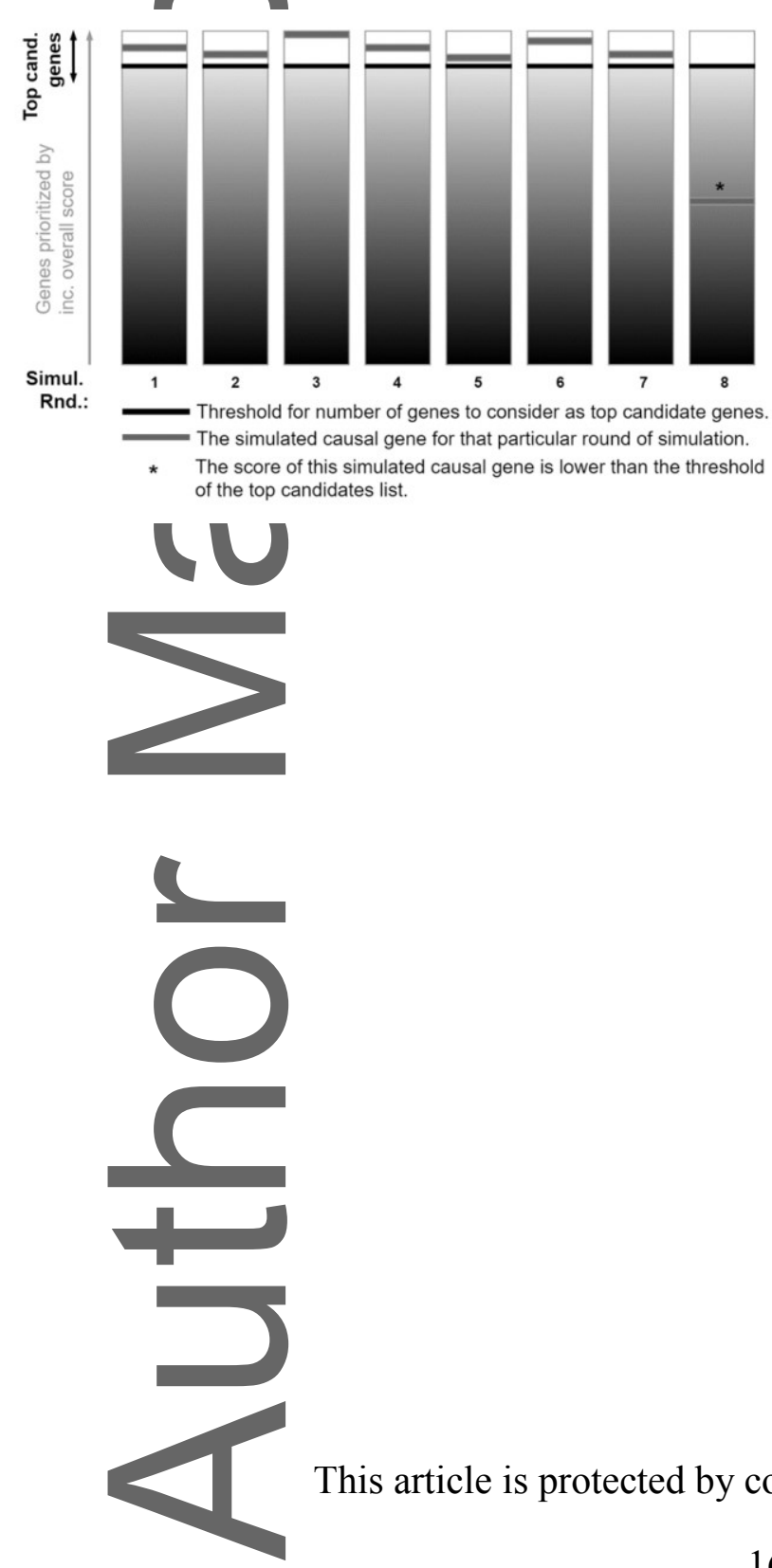

This article is protected by copyright. All rights reserved. 
Figure 2. Changes in estimated power across sample sizes, minimum pathogenicity scores, and penetrance.

The estimated power to prioritize the simulated causal gene(s) to the top candidates list in a proposed rare study design over different A) numbers of case-control pairings, B) minimum pathogenicity scores, and $\mathbf{C}$ ) levels of penetrance. The input causal gene pool consists of a subset of known autosomal recessive genes and compound heterozygosity has been flagged. All other parameters remain at default.
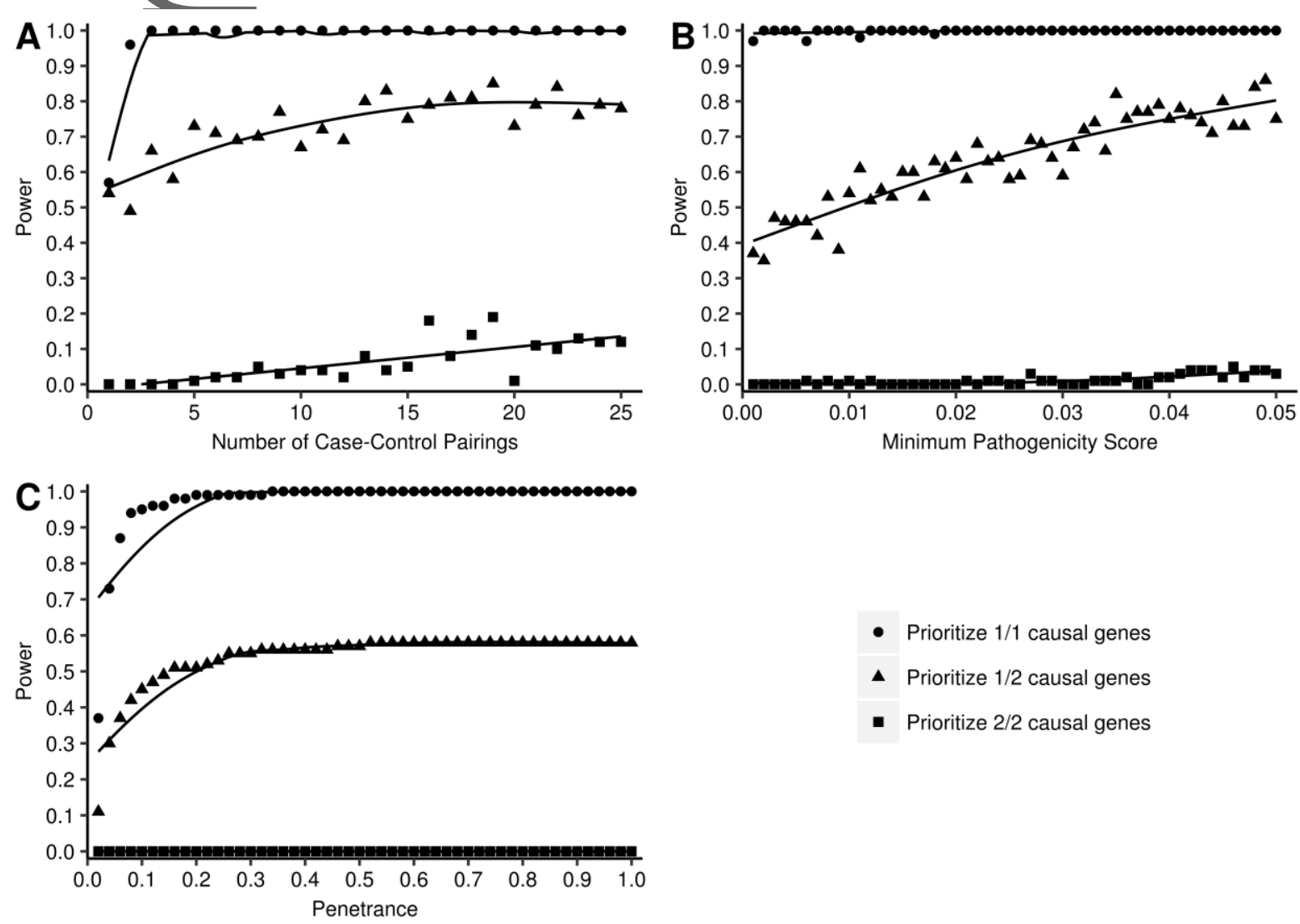

- Prioritize $1 / 1$ causal genes

- Prioritize 1/2 causal genes

- Prioritize 2/2 causal genes

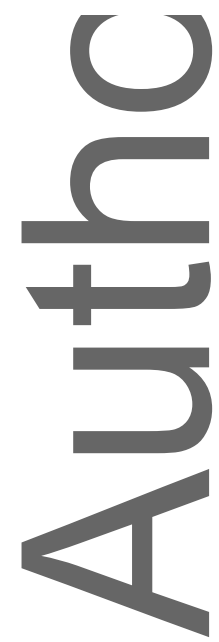

This article is protected by copyright. All rights reserved. 
Figure 3. Changes in estimated power across different numbers of parental controls.

The estimated power to prioritize the simulated causal gene(s) to the top candidates list in a proposed rare study design over different numbers of case-control pairings and different numbers of parental controls. The input causal gene pool consists of a subset of known autosomal recessive genes and compound heterozygosity has been flagged. All other parameters remain at default. A) The power to prioritize the causal gene where there is one causal gene for the lowprevalence condition. B) The power to prioritize one causal gene where there are two causal genes for the low-prevalence condition. C) The power to prioritize both causal genes where there are two causal genes for the low-prevalence condition.
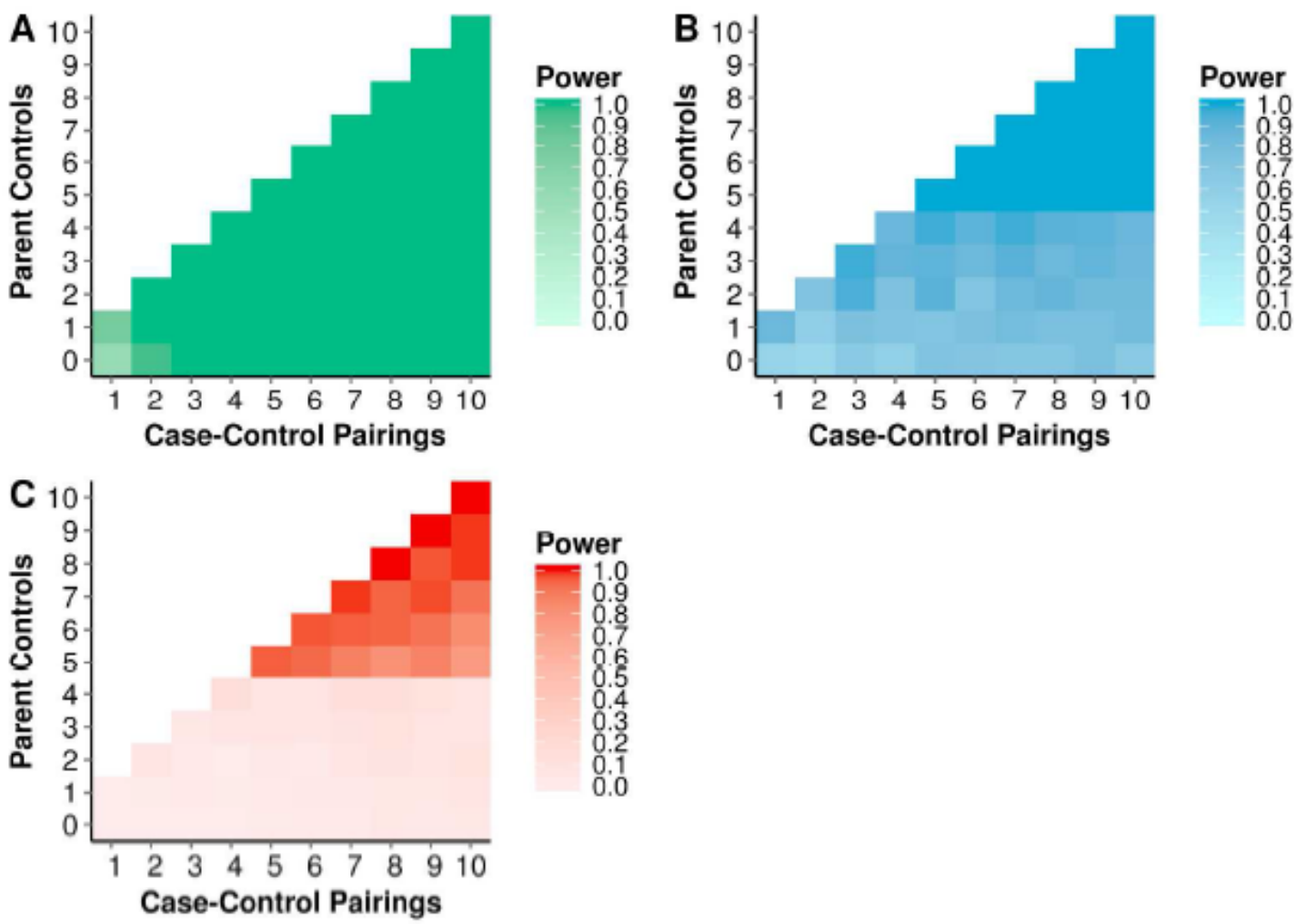

This article is protected by copyright. All rights reserved. 


\section{TABLES}

Table I. Key customizable SimPEL parameters and descriptions of function. Parameter

\section{Function}

\begin{tabular}{ll}
\hline Sample size & Number of case-control pairings simulated. \\
\hline Causal gene pool & A priori list of genes or model of inheritance. \\
\hline Number of causal genes & Heterogeneity of the simulated disease. \\
\hline Rank success list size & Affects FDR through included \# of genes. \\
\hline Prevalence/penetrance & Prevalence/penetrance of the focal condition. \\
\hline Confidence score & Pathogenicity score confidence. \\
\hline Score cutoff & Cutoff of the pathological score in simulation. \\
\hline Parents/siblings & Number of cases with matched familial controls. \\
\hline Compound heterozygosity & Consider compound heterozygous sites only.
\end{tabular}

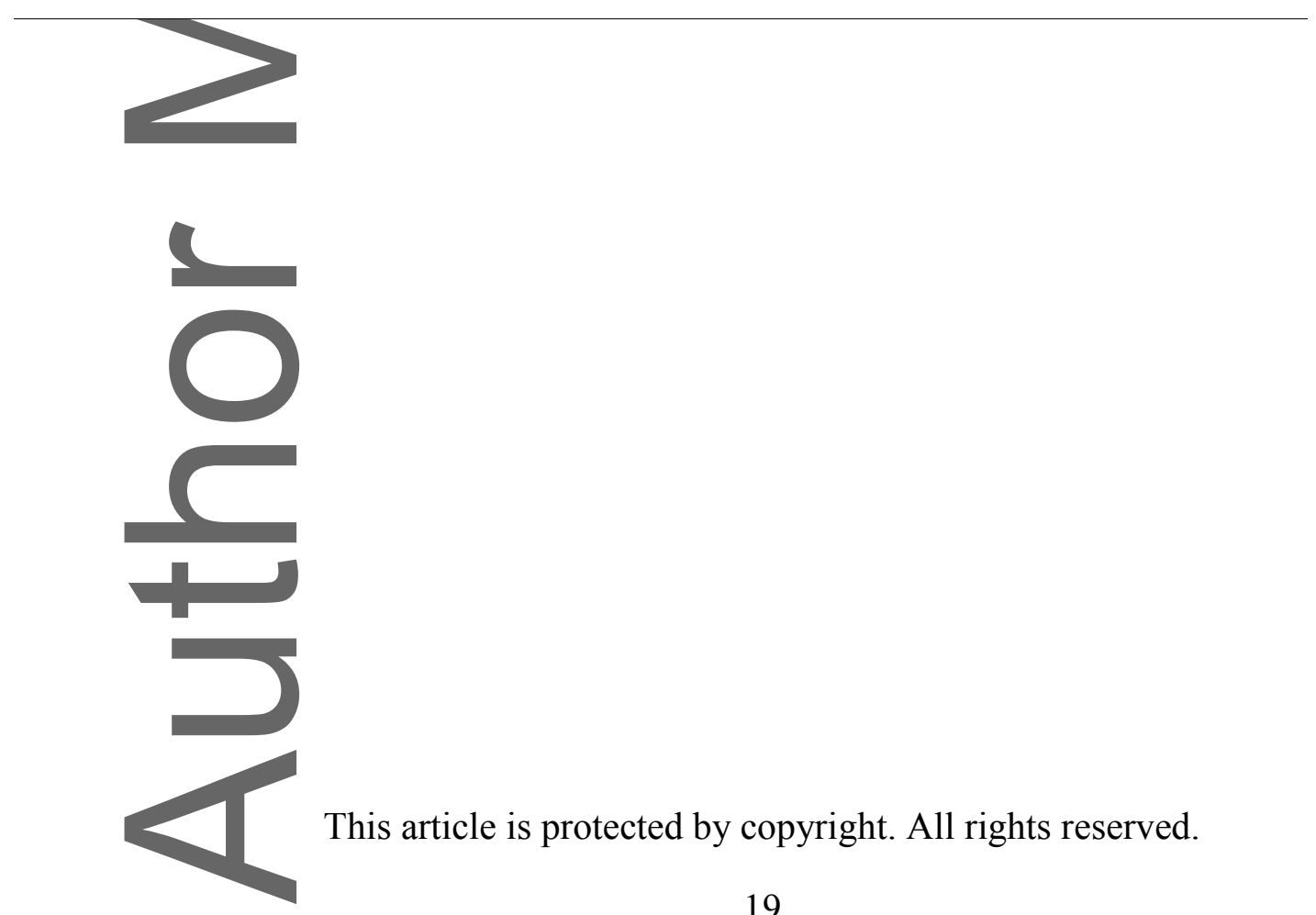




\section{University Library}

\section{- M M N E R VA A gateway to Melbourne's research publications}

Minerva Access is the Institutional Repository of The University of Melbourne

Author/s:

Mak, L;Li, M;Cao, C;Gordon, P;Tarailo-Graovac, M;Bousman, C;Wang, P;Long, Q

Title:

SimPEL: Simulation-based power estimation for sequencing studies of low-prevalence conditions

Date:

2018-07-01

Citation:

Mak, L., Li, M., Cao, C., Gordon, P., Tarailo-Graovac, M., Bousman, C., Wang, P. \& Long, Q. (2018). SimPEL: Simulation-based power estimation for sequencing studies of low-prevalence conditions. GENETIC EPIDEMIOLOGY, 42 (5), pp.480-487. https:// doi.org/10.1002/gepi.22129.

Persistent Link:

http://hdl.handle.net/11343/283983 